\title{
A Body Shape Index and Heart Rate Variability in Healthy Indians with Low Body Mass Index
}

\author{
Sharma Sowmya, ${ }^{1}$ Tinku Thomas, ${ }^{2}$ Ankalmadagu Venkatsubbareddy Bharathi, ${ }^{3}$ \\ and Sambashivaiah Sucharita ${ }^{1,3}$ \\ ${ }^{1}$ Department of Physiology, St. John's Medical College, Bangalore 560034, India \\ ${ }^{2}$ Division of Epidemiology and Statistics, St. John's Research Institute, Bangalore 560034, India \\ ${ }^{3}$ Clinical Physiology Unit, Department of Physiology, St. John's Medical College, Bangalore 560034, India
}

Correspondence should be addressed to Sambashivaiah Sucharita; sucharita.dr@gmail.com

Received 30 June 2014; Accepted 12 September 2014; Published 2 October 2014

Academic Editor: C. S. Johnston

Copyright (C) 2014 Sharma Sowmya et al. This is an open access article distributed under the Creative Commons Attribution License, which permits unrestricted use, distribution, and reproduction in any medium, provided the original work is properly cited.

Background. One third of Indian population is said to be suffering from chronic energy deficiency (CED), with increased risk of developing chronic diseases. A new anthropometric measure called A Body Shape Index (ABSI) is said to be a better index in predicting risks for premature mortality. ABSI is also in part said to be a surrogate of visceral fat. Objective. The present study aimed to explore the association between indices of HRV (heart rate variability), BMI, WC, and ABSI in healthy Indian males with low BMI $\left(\mathrm{BMI}<18.5 \mathrm{~kg} / \mathrm{m}^{2}\right.$ ) and to compare with normal BMI group (BMI 18.5 to $24.9 \mathrm{~kg} / \mathrm{m}^{2}$ ). Methodology. ABSI and BMI were derived from anthropometric parameters, namely, height, weight, and waist circumference in 178 males aged 18 to 78 years. Subjects were categorized into two groups based on their BMI. Results and Conclusions. Power spectral analysis of HRV demonstrated a significant negative correlation between Log HF (high frequency) and ABSI in both low BMI [-24.2 (9.4), $P<0.05]$ and normal BMI group [-23.41 (10.1), $P<0.05]$ even after controlling for age. Thus even with slight increase in BMI among low BMI individuals, there could be a greater risk of cardiovascular morbidity and mortality.

\section{Introduction}

Obesity has been the center of focus of majority of body composition related studies. This is partly due to a large body of data available from western population where obesity is considered a major public health concern [1-3]. Epidemiological studies with longitudinal follow-up have demonstrated association between simple anthropometric measures like height, weight (body mass index: BMI), and waist circumference (WC) with cardiovascular risks $[3,4]$. Within a given BMI range, association with increased cardiovascular risks becomes even stronger as WC increases [5]. Studies have linked alterations in autonomic nervous activity with increased cardiovascular risk [6]. Heart rate variability (HRV) is a simple noninvasive, sensitive measure to evaluate autonomic system activity. Changes in HRV have been demonstrated to detect even subclinical states $[7,8]$.
Decreased heart rate variability (both low and high frequency) has been observed in obese, compared to individuals with normal BMI [9-11]. Pockets of data available from Indian population are not different from those of western data $[12,13]$. However, India has an additional burden of chronic energy deficiency with one-third of population being of low BMI [14]. Recent evidence based on HRV measures from our laboratory demonstrated decreased HRV in undernourished subjects when compared to well-nourished/normal BMI group [15]. It has been speculated that undernourished/low BMI individuals might have more of visceral adiposity or ectopic fat than subcutaneous fat [16]. Although WC has been suggested as being superior to BMI in predicting cardiovascular risks in obese [17], its ability to predict increased cardiovascular risk in individuals with low BMI needs to be explored. Further, WC is highly sensitive to body size and fat distribution and is correlated with BMI. Thus, differentiating 
$\mathrm{BMI}$ and WC as two independent epidemiological risk factors becomes difficult. Recently a new anthropometric measure, called A Body Shape Index (ABSI), has been derived from waist circumference which is independent of BMI and is said to be a better index than using either WC or BMI independently. Thus being independent of BMI, ABSI helps in understanding whether abdominal obesity has predictive ability that cannot be explained by BMI alone. Current study aimed to explore the association between indices of HRV and the anthropometric measures including BMI, WC, and ABSI in healthy Indians with low BMI and compare it with normal.

\section{Materials and Methods}

One hundred and seventy eight males aged 18 to 78 years were recruited from in and around the medical college. Based on their BMI, subjects were categorized into low BMI group $\left(<18.5 \mathrm{~kg} / \mathrm{m}^{2}\right)$ and normal BMI group (between 18.5 and $\left.24.9 \mathrm{~kg} / \mathrm{m}^{2}\right)$. Subjects with any chronic ailments including diabetes, hypertension, and renal and endocrinal disorders were excluded. None of the subjects reported any symptoms suggestive of peripheral or autonomic neuropathy like giddiness on standing, urinary urgency, tingling sensation of limbs, or limb weakness or gastrointestinal symptoms like burning sensation in epigastric region, diarrhea, and constipation. The institutional ethical review board approved the research protocol.

All subjects underwent anthropometric assessment including weight recorded in minimal clothing to the nearest $0.1 \mathrm{~kg}$, using a digital scale (Alfoset, Model HW100KA1, Mumbai, India) and height being recorded to the nearest 0.1 centimeter, using a vertically mobile scale (Holtain, Crymych, UK). Body mass index was calculated as weight/height ${ }^{2}$. WC was measured using a standard nonstretchable tape measure, at the narrowest point between the iliac crest and ribcage. The measured WC from our population was compared with the predicted WC derived from the equation $\log (\mathrm{WC})=(-2.589 \pm 0.020)+(0.6807 \pm$ $0.0052) \log$ weight $-(0.814 \pm 0.020) \log$ height [18]. The measured WC was used for subsequent analysis. ABSI

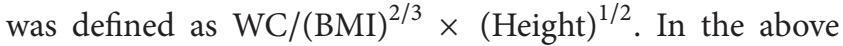
equation, weight was expressed in kilograms and height and WC in meters [18].

\subsection{Heart Rate Variability Assessment}

2.1.1. Preparation of the Subject. After explaining the process of ECG recording, subjects were asked to rest and relax in supine posture for 30 minutes. ECG recording was performed in a quiet room with soothing lighting and with comfortable temperature being maintained. Any electronic gadgets or metal or magnetic objects which could interfere with the ECG recording were removed. Skin was kept dry and rubbed with alcohol pad before application of electrodes.

2.1.2. Electrode Placement. Disposable electrodes were placed firmly onto the skin and we made sure that a good contact between the skin and the electrode was maintained. Electrodes were placed on the right arm, left arm, and left leg (lead II).

2.1.3. ECG Module. ECG module contains the electronics that detect electrical impulses from the heart and convert them into digital data that is transmitted to the computer. One end of the ECG module was connected to the subject through leads and another end to the USB port of the computer.

2.2. Processing of the ECG Signals. Analog lead II ECG signal obtained was digitized using analog to digital converter (CIOAD16Jr A/D card). Sampling frequency of ECG signals was $1000 \mathrm{~Hz}$ using an IBM compatible PC and a data acquisition package (CVMS; World Precision Instruments Inc., Sarasota, FL, USA.) The data acquisition system included a threshold peak detection system, from which RR intervals were determined. The RR intervals were plotted as a tachogram ( $R R$ values versus interval number). Data segments of $128 \mathrm{sec}$ duration were sampled at $2 \mathrm{~Hz}$ to create 256 point data sets. For each $10 \mathrm{~min}$ recording, an average of eight data sets of 256 points overlapping by half were processed. Heart rate variability analysis was performed using the frequency domain method. Power spectral density (PSD) was calculated using mathematical algorithm fast Fourier transform (FFT). The PSD was analyzed by calculating powers and peak frequencies for different frequency bands. No more than 3 data points were edited in a given segment [19].

2.2.1. Spectrum Estimation. FFT algorithm uses the signals in the time domain (RR interval) and transforms them into discrete frequency domain representation (power spectra). To prevent digital leakage during FFT Hanning digital filtering was used. The spectra obtained for the different data sets were averaged to reduce variance and to sharpen reproducible central peaks. Power was calculated in three bands. The 0.04$0.15 \mathrm{~Hz}$ band of RR power (referred to as the low-frequency band) reflects, at least in part, sympathetic nerve activity to the heart and partly parasympathetic activity, while the $0.15-$ $0.4 \mathrm{~Hz}$ band (high-frequency band) reflects parasympathetic nerve activity to the heart. Absolute total power (TP) represents the total variance of the power spectral density, that is, $0-0.4 \mathrm{~Hz}$ band $\left(\mathrm{msec}^{2}\right)$. In addition to the absolute power, data for heart rate variability are also presented as normalized units, as recommended [20], where the power in the low- and high-frequency bands are expressed as percentage of the total power minus the power of the very-low-frequency band (0.0$0.04 \mathrm{~Hz}$ ).

2.3. Statistical Analysis. The data was checked for normality using the Kolmogorov-Smirnov test. All normally distributed data are expressed as mean \pm SD and median (Quartile1 and Quartile3), when not normally distributed. The differences between low and normal BMI study group were compared using independent $t$ test. Bland-Altman plots [21] were constructed to examine limits of agreement between measured WC and the predicted WC. Heart rate variability indices were represented as log transformed (absolute units) 
TABLE 1: Descriptive characteristics of study population.

\begin{tabular}{|c|c|c|c|}
\hline Subject characteristics & $\begin{array}{l}\text { Entire group } \\
\quad(n=178)\end{array}$ & $\begin{array}{l}\text { Normal BMI group } \\
\quad(n=105)\end{array}$ & $\begin{array}{l}\text { Low BMI group } \\
\quad(n=73)\end{array}$ \\
\hline Age (Yr) & $40 \pm 22$ & $41 \pm 23$ & $35 \pm 22$ \\
\hline Weight (Kg) & $54.3 \pm 9.1$ & $59.3 \pm 7.9$ & $47.1 \pm 4.9$ \\
\hline Height (m) & $1.66 \pm 0.07$ & $1.66 \pm 0.08$ & $1.65 \pm 0.07$ \\
\hline Waist circumference (m) & $0.74 \pm 0.08$ & $0.79 \pm 0.01$ & $0.68 \pm 0.05^{*}$ \\
\hline $\mathrm{BMI}\left(\mathrm{Kg} / \mathrm{m}^{2}\right)$ & $19.6 \pm 2.6$ & $21.4 \pm 1.75$ & $17.08 \pm 0.9^{*}$ \\
\hline ABSI & $0.079 \pm 0.006$ & $0.079 \pm 0.006$ & $0.078 \pm 0.006$ \\
\hline Heart rate (bpm) & $63.1 \pm 9.9$ & $63.8 \pm 9.7$ & $62.1 \pm 10.3$ \\
\hline Low frequency power (LF) $\left(\mathrm{ms}^{2}\right)$ & $568.6(192.7-1155.9)$ & $569.1(196.1-1405.5)$ & $562.6(178.3-925.5)$ \\
\hline High frequency power $(\mathrm{HF})\left(\mathrm{ms}^{2}\right)$ & $538.4(175.7-1309.4)$ & $572.8(146.3-1396.1)$ & $522.8(220.7-872.1)$ \\
\hline Total power $(\mathrm{TP})\left(\mathrm{ms}^{2}\right)$ & $1843.9(718.6-3426.9)$ & $2055.9(685.1-4418.8)$ & $1617.6(90.3-2935)$ \\
\hline Low frequency $(\mathrm{nu})$ & $55.2(38.7-69.0)$ & $59.5(43.1-74.9)$ & $51.1(34.7-63.3)$ \\
\hline High frequency (nu) & $52.9(40.7-66.2)$ & $49.4(36.2-63.6)$ & $55.5(44.9-70.7)$ \\
\hline LF nu/HF nu ratio & $1.1(0.6-1.6)$ & $1.2(0.7-1.9)$ & $0.9(0.5-1.5)$ \\
\hline
\end{tabular}

Grouping based on BMI ranges $\geq 18.5$ and $<25$ as normal BMI group and BMI $<18.5$ as low BMI group.

Data are mean \pm standard deviation/median (interquartile range), ${ }^{*} P<0.05$.

data. Correlation of log transformed HRV indices with BMI, WC, and ABSI was examined using Pearson's correlation coefficient. Further, correlation of HRV indices with BMI, $\mathrm{WC}$, and ABSI was examined in low BMI and normal BMI study groups. The correlation of HRV with age, ABSI, and WC was examined using linear regression models in the two BMI groups. Statistical significance was considered at $P<0.05$. All statistical analyses were performed using SPSS v18, SPSS, Chicago, IL.

\section{Results}

Table 1 represents the anthropometric and HRV indices in absolute units. Out of the 178 subjects, based on their BMI, 73 subjects were categorized as low BMI group and 105 subjects as normal BMI group. Predicted WC was within limits of agreement when compared with measured WC. Mean of difference between observed WC from the study population and predicted WC was -0.01 and the limits of agreement were 0.10 and -0.12 (Figure 1 ). BMI was positively and significantly correlated with WC $(r=0.77, P<0.001)$ and not with ABSI $(r=0.028, P=0.71)$. HRV indices were significantly and negatively correlated with WC $(\log \mathrm{LF}, r=-0.25, P<0.01$; $\log$ HF, $r=-0.30, P<0.01$; $\log$ TP, $r=-0.22, P<0.01$ ) and ABSI $(\log \mathrm{LF}, r=-0.54, P<0.01 ; \log \mathrm{HF}, r=-0.59$, $P<0.01$; $\log$ TP, $r=-0.55, P<0.01)$ but not with BMI ( $\log \mathrm{LF}, r=0.06, P=0.4 ; \log \mathrm{HF}, r=0.03, P=0.7 ; \log \mathrm{TP}$, $r=0.1, P=0.2$ ). The correlation of ABSI with HRV remained significant even after controlling for age but not that of WC with HRV (Figure 2). When this correlation was examined separately within low BMI and normal BMI groups, ABSI was significantly correlated with all HRV indices in the normal group after controlling for age, whereas Log HF alone was correlated with ABSI in the low BMI group (Table 2). None of the HRV indices were correlated with WC in either of the groups after controlling for age. There was no significant

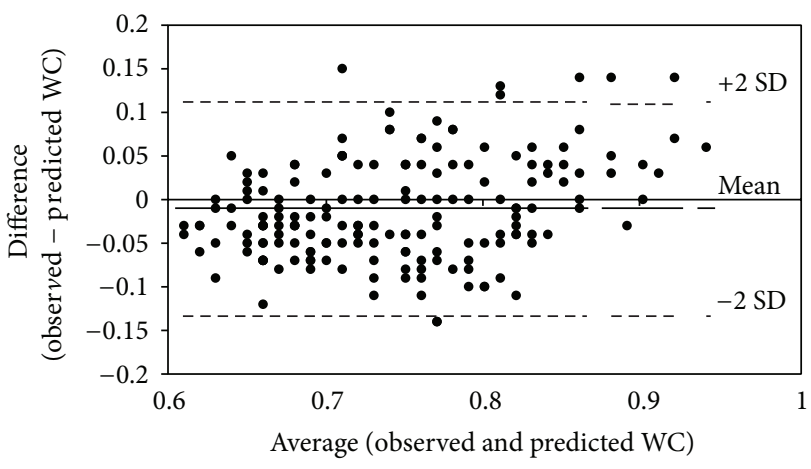

FIGURE 1: Bland Altman plot of WC differences between observed and predicted values. The solid line is at the bias and the dashed lines are at $\pm 95 \%$ limits of agreement.

correlation between the normalized values (nu of LF and HF) and ABSI, BMI or WC.

\section{Discussion}

Current study demonstrated that ABSI was significantly and negatively correlated with LF, HF, and TP indices of HRV. When grouped based on BMI, HF (an indicator of cardiac PNS activity) was negatively and significantly correlated with ABSI in both low BMI and normal BMI study group. However, LF (by far an indicator of cardiac SNS activity) and TP (total variability) were negatively correlated with ABSI only in normal BMI study group.

Nutritional transition is one of the major concerns in India [22]. Increased migration of individuals from rural to urban setup and increased urbanization has caused easy access to high caloric foods [23]. This has caused individuals with low BMI to shift towards either becoming normal BMI or even obese [24]. With reduced physical activity and 



FIGURE 2: Scatter plots representing the partial correlation between BMI, WC, ABSI, and HRV after adjusting for age. Variables are represented after adjusting for age. ${ }^{*} P<0.05$ is considered statistically significant. Correlations without adjusting for age are represented in the results.

TABLE 2: Linear regression models describing the associations between HRV indices versus ABSI and WC in normal BMI and low BMI study groups.

\begin{tabular}{|c|c|c|c|c|c|c|c|c|}
\hline \multirow[t]{2}{*}{ Linear regression } & & & \multicolumn{2}{|c|}{$\begin{array}{l}\log \mathrm{LF} \\
\beta(\operatorname{Se} \beta)\end{array}$} & \multicolumn{2}{|c|}{$\begin{array}{l}\log \mathrm{HF} \\
\beta(\operatorname{Se} \beta)\end{array}$} & \multicolumn{2}{|c|}{$\begin{array}{l}\log \mathrm{TP} \\
\beta(\operatorname{Se} \beta)\end{array}$} \\
\hline & & & Low BMI & Normal BMI & Low BMI & Normal BMI & Low BMI & Normal BMI \\
\hline \multirow{3}{*}{ ABSI } & Model 1 & ABSI & $-43.15(8.5)^{*}$ & $-46.47(7.1)^{*}$ & $-47.51(7.7)^{*}$ & $-63.93(8.6)^{*}$ & $-37.33(7.3)^{*}$ & $-48.61(6.9)^{*}$ \\
\hline & \multirow{2}{*}{ Model 2} & ABSI & $-13.73(10.1)$ & $-21.73(8.9)^{*}$ & $-24.20(9.4)^{*}$ & $-23.41(10.1)^{*}$ & $-15.60(8.9)$ & $-20.33(8.4)^{*}$ \\
\hline & & AGE & $-0.01(0.0)^{*}$ & $-0.01(0.0)^{*}$ & $-0.01(0.0)^{*}$ & $0.02(0.0)^{*}$ & $-0.01(0.0)^{*}$ & $-0.01(0.0)^{*}$ \\
\hline \multirow{3}{*}{ WC } & Model 1 & WC & $-0.36(0.0)^{*}$ & $-0.02(0.0)^{*}$ & $-0.04(0.0)^{*}$ & $-0.03(0.0)^{*}$ & $-0.03(0.0)^{*}$ & $-0.02(0.0)^{*}$ \\
\hline & \multirow{2}{*}{ Model 2} & WC & $-0.01(0.0)$ & $-0.01(0.0)$ & $-0.02(0.0)$ & $-0.01(0.0)$ & $-0.01(0.0)$ & $-0.01(0.0)$ \\
\hline & & AGE & $-0.01(0.0)^{*}$ & $-0.01(0.0)^{*}$ & $-0.01(0.0)^{*}$ & $-0.02(0.0)^{*}$ & $-0.01(0.0)^{*}$ & $-0.01(0.0)^{*}$ \\
\hline
\end{tabular}

Model 1 represents either ABSI or WC. Model 2 represents either ABSI or WC after controlling for age; values represent regression coefficient $\beta$ (standard error of $\beta)^{*} P<0.05$.

increased caloric consumption with preexisting low muscle mass, susceptibility to develop chronic disease is increasing in Indians [22-24]. One of the contributing factors is increased body fat accumulation, particularly fat within tissues, which is commonly termed as ectopic/visceral fat. The visceral deposition of fat is considered to be critical in defining the risk for metabolic disease [25] and is relatively more pronounced in Asian Indians compared to other ethnic groups [26]. Visceral fat carries greater risk of insulin resistance, CVS disease, metabolic syndrome $[27,28]$.

Measures of visceral fat are difficult, tedious and require high-end instruments [29]. Although these techniques might 
help us give accurate measures, their feasibility and application at a population level are questionable. Simple indices like WC have long been used as a surrogate of central adiposity [30]. Using WC, a simple index called ABSI has emerged of late, which could by far act as a surrogate of visceral fat [18]. In this study there was a good agreement between measured and predicted WC. This allowed us to utilize WC in deriving ABSI for Indian population.

Increasing central adiposity (measured using waist circumference) has been associated with a reduced autonomic functionality [17]. This has been demonstrated in individuals who are obese [31] or who are on a weight loss program [32]. There is lack of understanding of association between indices of central or visceral adiposity and autonomic nervous activity particularly in individuals with low-normal BMI.

Reduced autonomic function predominantly parasympathetic nervous activity has been linked to visceral fat [33]. Christou et al. reported higher abdominal-to-peripheral body fat distributions measured by DXA were strongly correlated with lower sympathetic and parasympathetic function in young and old healthy men [34]. Other studies have also shown that body fat distribution is a major factor, particularly visceral fat carrying the greatest risk for cardiovascular morbidity and mortality $[26,27,35]$. In keeping with this, data from the current study demonstrated that cardiac autonomic activity particularly HF (cardiac parasympathetic activity) was negatively correlated with ABSI across BMI range. This suggests that one of the possible explanations for reduced cardiac parasympathetic activity could well be due to increased ABSI which is by far an indicator of visceral fat.

Greater degree of autonomic involvement, that is, both cardiac sympathetic and parasympathetic nervous activity, is suggested to be associated with higher visceral adiposity $[10,34]$. The fact that normal BMI group were having significantly greater WC and marginally higher ABSI though not significant suggests the possibility of greater visceral adiposity in this group. In addition, data from the current study demonstrated a negative association with cardiac sympathetic activity and ABSI in the normal study group. One of the mechanisms which has been linked to greater autonomic involvement (both components of ANS) and visceral fat includes reduced arterial distensibility [36]. Carotid artery distensibility is an important physiological determinant of cardiovagal baroreflex gain [37]. Therefore, it is possible that lower levels of carotid artery distensibility with elevated visceral fat would reduce mechanical transduction of arterial blood pressure into barosensory stretch [33]. Another mechanism could be greater circulating neurohumoural factors that act centrally modifying the cardiovagal baroreflex $[38,39]$. Finally, impaired muscarinic receptor function has been demonstrated in an animal model of dietinduced obesity [40]. Thus it is possible that the reduction in cardiovagal baroreflex gain observed with elevated total body and abdominal visceral fat in the present study was due, in part, to reduced cardiac muscarinic receptor number and/or sensitivity.

Thin-fat phenotype, a term coined for Indian population with low-normal BMI, might well have greater visceral adiposity. This calls for urgent need to explore the visceral adiposity status in Indians across all BMI ranges. Possible causes for greater deposition of fat at tissue level may well be due to states of positive energy balance, environmental exposures such as inflammation, stress, tobacco, alcohol, and pollution interacting, and amplifying the effect of the deposition of fat on a thin frame [24]. Studies are further required to explore the role of above factors in contributing towards the deposition of visceral adiposity. Further, studies are also required to explore implication of ABSI in larger population based follow-up studies including females to understand the role of ABSI in predicting the metabolic risk in low BMI group.

In conclusion, greater ABSI by far an indicator of visceral adiposity was associated with reduced cardiac parasympathetic and sympathetic activity even in individuals with low BMI. Thus, even with slight increase in BMI among low BMI individuals, there could be a greater risk of cardiovascular morbidity and mortality.

\section{Conflict of Interests}

The authors declare that there is no conflict of interests regarding the publication of this paper.

\section{Acknowledgment}

Part of the data presented in the current study was funded by Nestle Foundation, Switzerland.

\section{References}

[1] D. B. Allison, K. R. Fontaine, J. E. Manson, J. Stevens, and T. B. VanItallie, "Annual deaths attributable to obesity in the United States," The Journal of the American Medical Association, vol. 282, no. 16, pp. 1530-1538, 1999.

[2] M. Duncan, M. Griffith, H. Rutter, and M. J. Goldacre, "Certification of obesity as a cause of death in England 1979-2006," European Journal of Public Health, vol. 20, no. 6, pp. 671-675, 2010.

[3] T. Pischon, H. Boeing, K. Hoffmann et al., "General and abdominal adiposity and risk of death in Europe," The New England Journal of Medicine, vol. 359, no. 20, pp. 2105-2120, 2008.

[4] A. B. Gonzalez, P. Hartge, J. R. Cerhan et al., "Body-Mass Index and mortality among 1.46 million white adults," New England Journal of Medicine, vol. 363, pp. 2211-2219, 2010.

[5] J. Bigaard, A. Tjønneland, B. L. Thomsen, K. Overvad, B. L. Heitmann, and T. I. A. Sørensen, "Waist circumference, BMI, smoking, and mortality in middle-aged men and women," Obesity Research, vol. 11, no. 7, pp. 895-903, 2003.

[6] B. M. Curtis and J. H. O'Keefe, "Autonomic tone as a cardiovascular risk factor: the dangers of chronic fight or flight," Mayo Clinic Proceedings, vol. 77, no. 1, pp. 45-54, 2002.

[7] D. Ziegler, "Diabetic cardiovascular autonomic neuropathy: prognosis, diagnosis and treatment," Diabetes/Metabolism Reviews, vol. 10, no. 4, pp. 339-383, 1994.

[8] A. I. Vinik, R. E. Maser, B. D. Mitchell, and R. Freeman, "Diabetic autonomic neuropathy," Diabetes Care, vol. 26, no. 5, pp. 1553-1579, 2003. 
[9] B. Zahorska-Markiewicz, L. E. Kuagowska, C. Kucio, and M. Klin, "Heart rate variability in obesity," International Journal of Obesity, vol. 17, no. 1, pp. 21-23, 1993.

[10] K. Laederach-Hofmann, L. Mussgay, and H. Rúddel, "Autonomic cardiovascular regulation in obesity," Journal of Endocrinology, vol. 164, no. 1, pp. 59-66, 2000.

[11] H. R. Peterson, M. Rothschild, C. R. Weinberg, R. D. Fell, K. R. McLeish, and M. A. Pfeifer, "Body fat and the activity of the autonomic nervous sytem," The New England Journal of Medicine, vol. 318, no. 17, pp. 1077-1083, 1988.

[12] A. R. Shenoy, V. Doreswamy, J. P. Shenoy, and V. S. Prakash, "Impact of obesity on cardiac autonomic functions in middle aged males," National Journal of Physiology Pharmacy and Pharmacology, vol. 4, no. 3, 2014.

[13] R. Rajalakshmi, Y. VijayaVageesh, S. M. Nataraj, MuraliDhar, and C. G. Srinath, "Heart rate variability in Indian obese young adults," Pakistan Journal of Physiology, vol. 8, no. 1, pp. 39-44, 2012.

[14] Indian Institute of Population Sciences, Mumbai, India, National Family Health Survey-3, vol. 1, pp. 303-313, 2006.

[15] M. Vaz, A. V. Bharathi, S. Sucharita, and D. Nazareth, "Heart rate variability and baroreflex sensitivity are reduced in chronically undernourished, but otherwise healthy, human subjects," Clinical Science, vol. 104, no. 3, pp. 295-302, 2003.

[16] A. Misra and L. Khurana, "Obesity-related non-communicable diseases: South Asians vs White Caucasians," International Journal of Obesity, vol. 35, no. 2, pp. 167-187, 2011.

[17] B. G. Windham, S. Fumagalli, A. Ble et al., "The relationship between heart rate variability and adiposity differs for central and overall adiposity," Journal of Obesity, vol. 2012, Article ID 149516, 8 pages, 2012.

[18] N. Y. Krakauer and J. C. Krakauer, "A new body shape index predicts mortality hazard independently of body mass index," PLoS ONE, vol. 7, no. 7, Article ID e39504, 2012.

[19] S. Sucharita, A. V. Bharathi, A. V. Kurpad, and M. Vaz, "A comparative study of tests of cardiac parasympathetic nervous activity in healthy human subjects," Physiological Measurement, vol. 23, no. 2, pp. 347-354, 2002.

[20] "Heart rate variability: standards of measurement, physiological interpretation and clinical use. Task Force of the European Society of Cardiology and the North American Society of Pacing and Electrophysiology," Circulation, vol. 93, no. 5, pp. 1043-1065, 1996.

[21] J. M. Bland and D. G. Altman, "Statistical methods for assessing agreement between two methods of clinical measurement," Lancet, vol. 1, no. 8476, pp. 307-310, 1986.

[22] P. S. Shetty, "Nutrition transition in India," Public Health Nutrition, vol. 5, no. 1, pp. 175-182, 2002.

[23] L. Bowen, S. Ebrahim, B. de Stavola et al., "Dietary intake and rural-urban migration in India: a cross-sectional study," PLoS ONE, vol. 6, no. 6, Article ID e14822, 2011.

[24] A. V. Kurpad, K. S. Varadharajan, and I. Aeberli, "The thin-fat phenotype and global metabolic disease risk," Current Opinion in Clinical Nutrition and Metabolic Care, vol. 14, no. 6, pp. 542547, 2011.

[25] K. P. McMillan, J. L. Kuk, T. S. Church, S. N. Blair, and R. Ross, "Independent associations between liver fat, visceral adipose tissue, and metabolic risk factors in men," Applied Physiology, Nutrition and Metabolism, vol. 32, no. 2, pp. 265-272, 2007.

[26] S. A. Lear, K. H. Humphries, S. Kohli, A. Chockalingam, J. J. Frohlich, and C. L. Birmingham, "Visceral adipose tissue accumulation differs according to ethnic background: results of the Multicultural Community Health Assessment Trial (MCHAT)," The American Journal of Clinical Nutrition, vol. 86, no. 2, pp. 353-359, 2007.

[27] M. A. Banerji, N. Faridi, R. Atluri, R. L. Chaiken, and H. E. Lebovitz, "Body composition, visceral fat, leptin, and insulin resistance in Asian Indian men," The Journal of Clinical Endocrinology and Metabolism, vol. 84, no. 1, pp. 137-144, 1999.

[28] G. A. Rosito, J. M. Massaro, U. Hoffmann et al., "Pericardial fat, visceral abdominal fat, cardiovascular disease risk factors, and vascular calcification in a community-based sample the framingham heart study," Circulation, vol. 117, no. 5, pp. 605613, 2008.

[29] K. van der Kooy and J. C. Seidell, "Techniques for the measurement of visceral fat: a practical guide," International Journal of Obesity, vol. 17, no. 4, pp. 187-196, 1993.

[30] M.-C. Pouliot, J.-P. Després, S. Lemieux et al., "Waist circumference and abdominal sagittal diameter: best simple anthropometric indexes of abdominal visceral adipose tissue accumulation and related cardiovascular risk in men and women," The American Journal of Cardiology, vol. 73, no. 7, pp. 460-468, 1994.

[31] A. Damodaran and B. Kabali, "Autonomic dysfunction in central obesity," World Journal of Medical Sciences, vol. 8, no. 2, pp. 118-122, 2013.

[32] G. E. Alvarez, B. M. Davy, T. P. Ballard, S. D. Beske, and K. P. Davy, "Weight loss increases cardiovagal baroreflex function in obese young and older men," American Journal of PhysiologyEndocrinology and Metabolism, vol. 289, no. 4, pp. E665-E669, 2005.

[33] S. D. Beske, G. E. Alvarez, T. P. Ballard, and K. P. Davy, "Reduced cardiovagal baroreflex gain in visceral obesity: implications for the metabolic syndrome," The American Journal of PhysiologyHeart and Circulatory Physiology, vol. 282, no. 2, pp. H630H635, 2002.

[34] D. D. Christou, P. P. Jones, A. E. Pimentel, and D. R. Seals, "Increased abdominal-to-peripheral fat distribution contributes to altered autonomic-circulatory control with human aging," The American Journal of Physiology-Heart and Circulatory Physiology, vol. 287, no. 4, pp. H1530-H1537, 2004.

[35] B. Larsson, "Regional obesity as a health hazard in menprospective studies," Acta Medica Scandinavica, vol. 223, no. 723, pp. 45-51, 1988.

[36] L. M. Resnick, D. Militianu, A. J. Cunnings, J. G. Pipe, J. L. Evelhoch, and R. L. Soulen, "Direct magnetic resonance determination of aortic distensibility in essential hypertension: relation to age, abdominal visceral fat, and in situ intracellular free magnesium," Hypertension, vol. 30, no. 3, pp. 654-659, 1997.

[37] I. Bonyhay, G. Jokkel, and M. Kollai, "Relation between baroreflex sensitivity and carotid artery elasticity in healthy humans," The American Journal of Physiology-Heart and Circulatory Physiology, vol. 271, no. 3, pp. H1139-H1144, 1996.

[38] S. Engeli and A. M. Sharma, "Role of adipose tissue for cardiovascular-renal regulation in health and disease," Hormone and Metabolic Research, vol. 32, no. 11-12, pp. 485-499, 2000.

[39] V. van Harmelen, M. Elizalde, P. Ariapart et al., “The association of human adipose angiotensinogen gene expression with abdominal fat distribution in obesity," International Journal of Obesity, vol. 24, no. 6, pp. 673-678, 2000.

[40] M. Pelat, P. Verwaerde, C. Merial et al., "Impaired atrial M2cholinoceptor function in obesity-related hypertension," Hypertension, vol. 34, no. 5, pp. 1066-1072, 1999. 


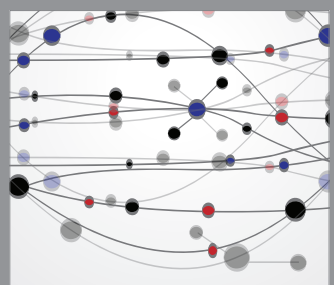

The Scientific World Journal
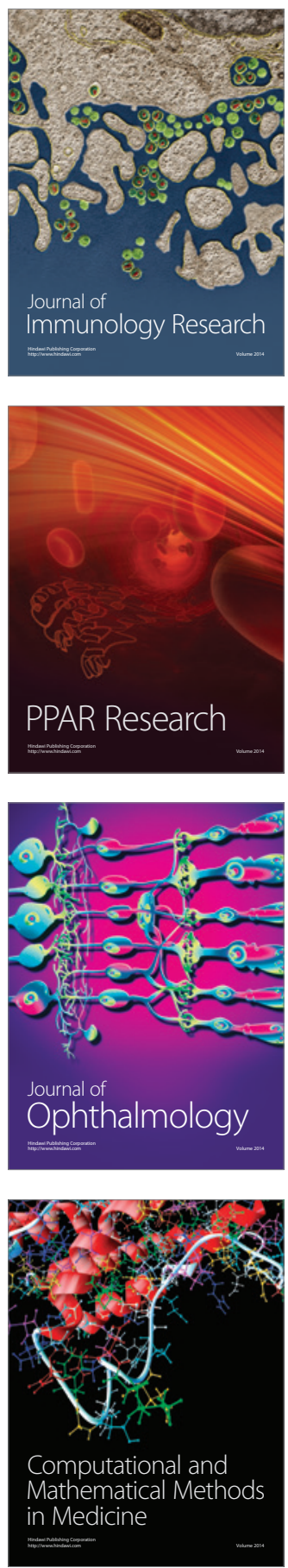

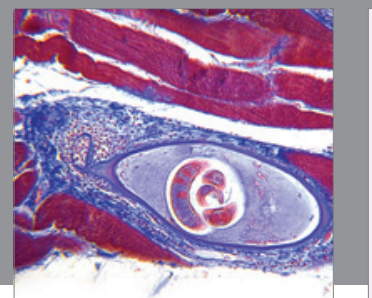

Gastroenterology

Research and Practice
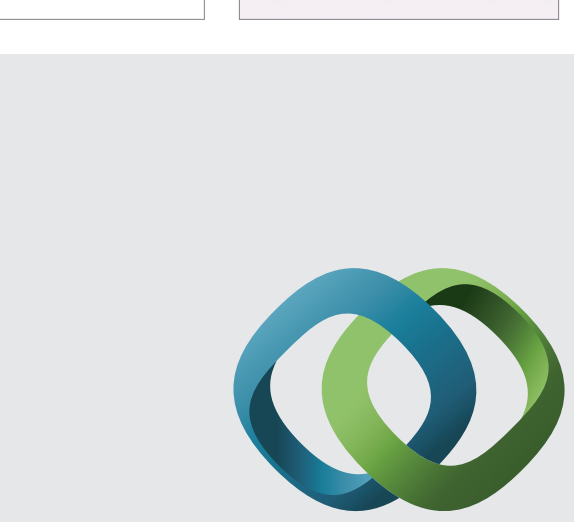

\section{Hindawi}

Submit your manuscripts at

http://www.hindawi.com
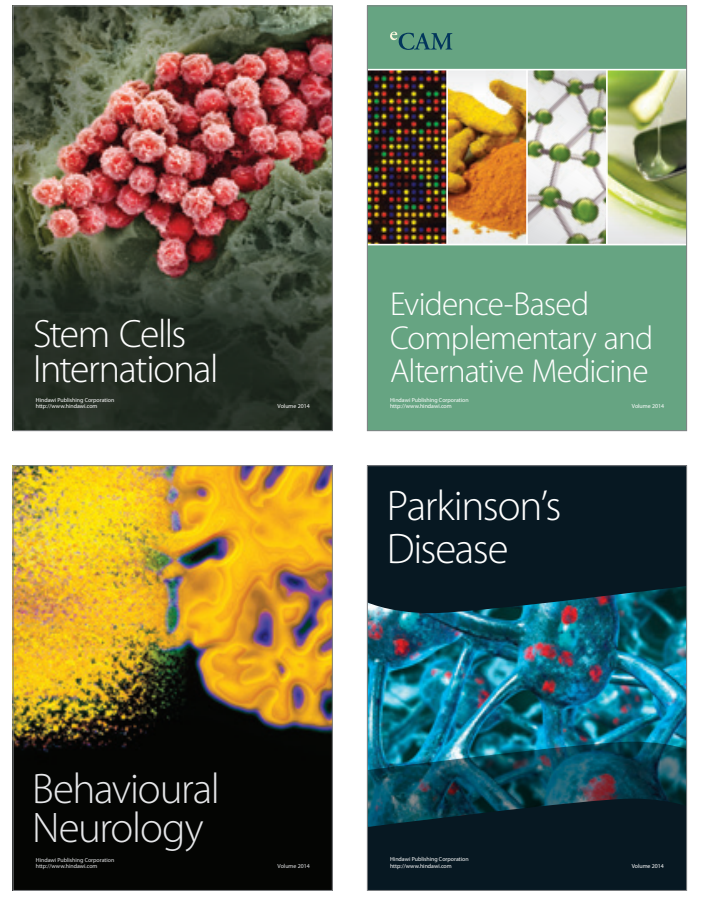
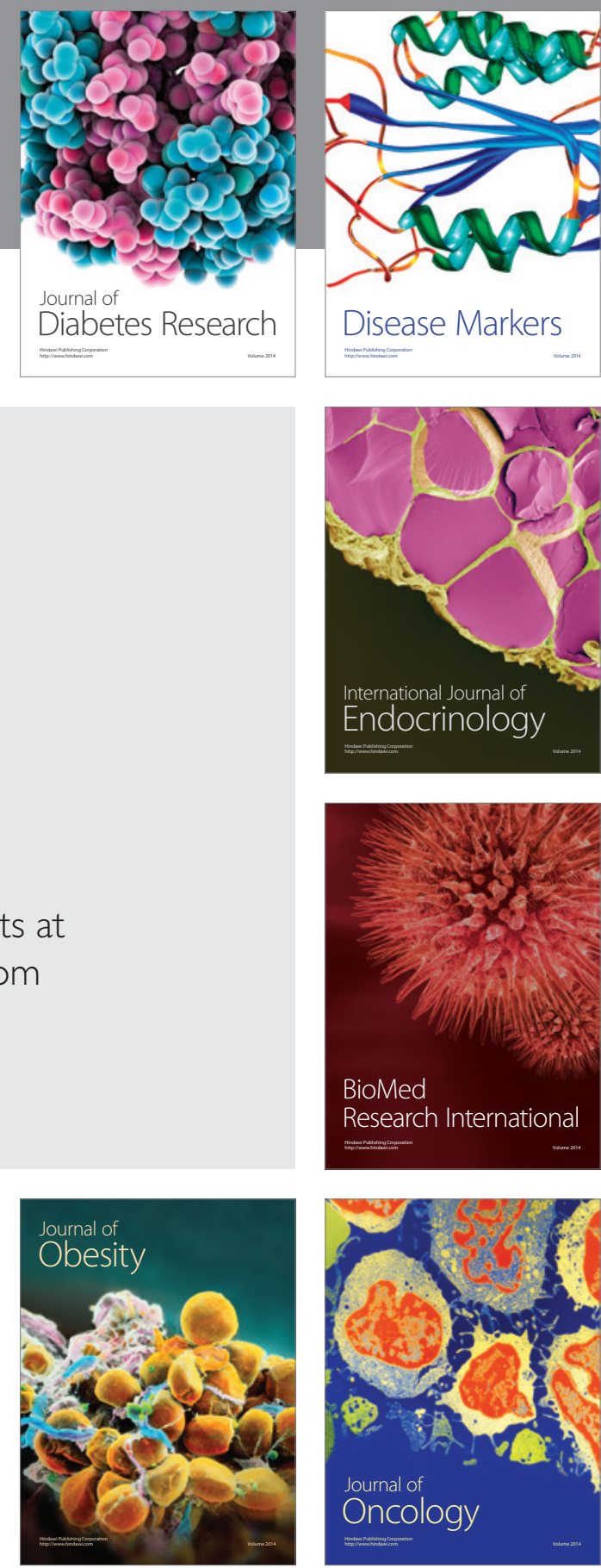

Disease Markers
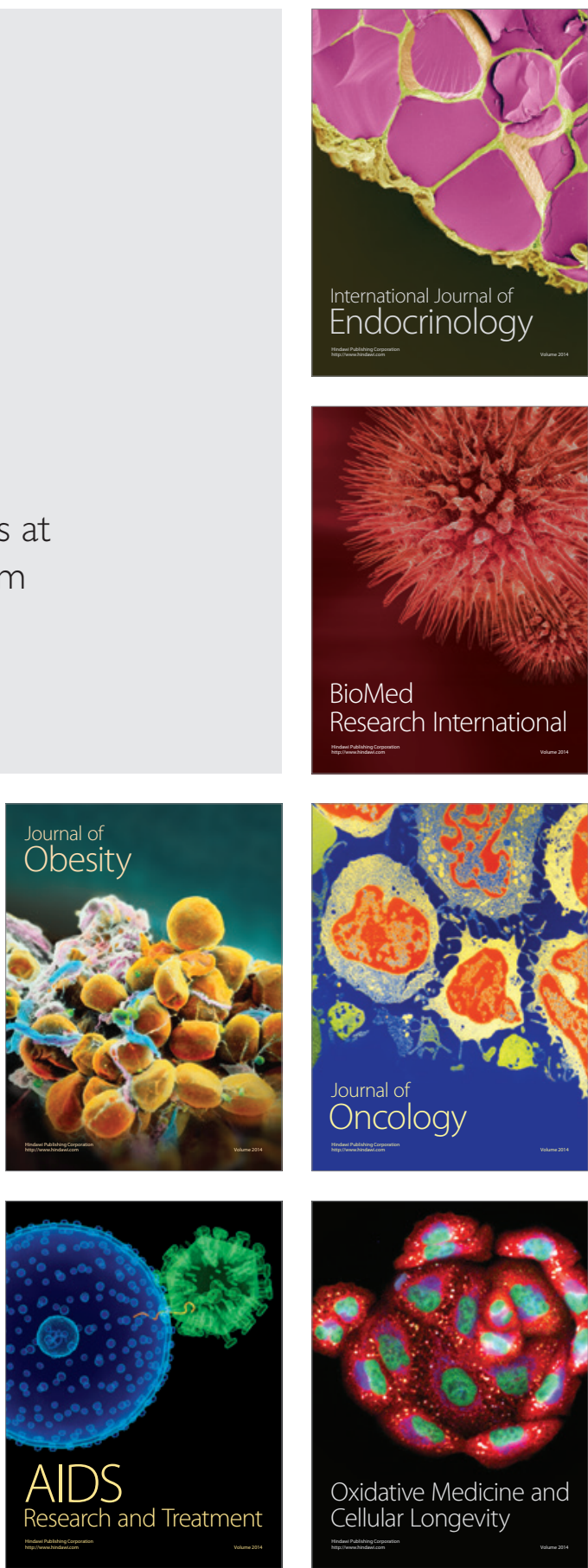